\title{
On Characterization of Gompertz Distribution by Properties of Generalized Record Values
}

\author{
S. Minimol ${ }^{1}$ and P. Yageen Thomas ${ }^{2}$ \\ Department of Statistics \\ University of Kerala, Trivandrum \\ 1 minibhama@ rediffmail.com \\ 2 yageenthomas@gmail.com
}

Received 2 December 2012

Accepted 31 December 2013

\begin{abstract}
In this chapter, we derive some recurrence relations satisfied by single and product moments of generalized record values arising from Gompertz and inverted Gompertz distributions. Further, we have obtained characterization of Gompertz and inverted Gompertz distributions based on certain properties of generalized record values.

Keywords: generalized record values, $k$-th record values, recurrence relation, Gompertz distribution, inverted Gompertz distribution, characterization of distribution.
\end{abstract}

AMS 2000 Subject Classification: 62E10, 62G99.

\section{Introduction}

The statistical study of record values in a sequence of iid continuous random variables was first formulated by Chandler (1952). For an extensive study in this area one can refer to the works of Arnold et al. (1998) and Ahsanullah (1988, 1995). Dzuibdziela and Kopocinski (1976) have generalized the concept of record values of Chandler (1952) by random variables of a more generalized nature and we may call them as generalized record values or $k$-th record values. In this chapter we mainly focus on the study of generalized record values from Gompertz distribution.

The Gompertz distribution was introduced by Gompertz (1825). This distribution plays an important role in modelling human mortality and fitting actuarial tables. Ahuja and Nash (1967) have shown that Gompertz distribution is related by a simple transformation to certain distributions in the Pearson system of distribution. It can be viewed as extensions of the exponential distributions. More recent survey of Gompertz distribution and its applications can be found in Al-Hussaini et al. (2000) and Marshall and Olkin (2007).

Let $\left\{X_{n}, n \geqslant 1\right\}$ be a sequence of iid random variables with cumulative distribution function (cdf) $F(x)=P(X \leqslant x)$ and probability density function (pdf) $f(x)$. For a fixed positive integer $k$, 
we define the sequence $\left\{U_{m}^{(k)}, m \geqslant 1\right\}$ of $k$-th upper record times of $\left\{X_{n}, n \geqslant 1\right\}$ as follows:

$$
U_{1}^{(k)}=1, \quad U_{m+1}^{(k)}=\min \left\{j>U_{m}^{(k)}: X_{j: j+k-1}>X_{U_{m}^{(k)}: U_{m}^{(k)}+k-1}\right\} .
$$

The sequence $\left\{Y_{m}^{(k)}, m \geqslant 1\right\}$ where $Y_{m}^{(k)}=X_{U_{m}^{(k)}: U_{m}^{(k)}+k-1}$ is called the sequence of $k$-th upper record values or generalized upper record values of $\left\{X_{n}, n \geqslant 1\right\}$. For convenience we shall also take $Y_{0}^{(k)}=$ 0 . For $k=1$ and $m=1,2, \ldots$ we write $U_{m}^{(1)}=U_{m}$. Then $\left\{U_{m}, m \geqslant 1\right\}$ is the sequence of upper record times of $\left\{X_{n}, n \geqslant 1\right\}$ as defined in Chandler (1952). We shall write,

$$
\begin{array}{lll}
\mu_{m ; k}^{(r)}=E\left[\left(Y_{m}^{(k)}\right)^{r}\right], & r, m=1,2, \ldots & \\
\mu_{m, t ; k}^{(r, s)}=E\left[\left(Y_{m}^{(k)}\right)^{r}\left(Y_{t}^{(k)}\right)^{s}\right], & 1 \leqslant m \leqslant t-1, & r, s=1,2, \ldots \\
\mu_{m, t ; k}^{(r, 0)}=E\left[\left(Y_{m}^{(k)}\right)^{r}\right]=\mu_{m ; k}^{(r)}, & 1 \leqslant m \leqslant t-1, & r=1,2, \ldots \\
\mu_{m, t ; k}^{(0, s)}=E\left[\left(Y_{t}^{(k)}\right)^{s}\right]=\mu_{t ; k}^{(s)}, & 1 \leqslant m \leqslant t-1, & s=1,2, \ldots
\end{array}
$$

In Section 2, we derive recurrence relations for single and product moments of generalized upper record values arising from Gompertz distribution. In Section 3, we derive the conditional distribution of a generalized upper record value $Y_{t}^{(k)}$ given $Y_{m}^{(k)}=x$ for $m<t$ and used a property of conditional expectation of a function of generalized upper record values to characterize Gompertz distribution. In Section 4, we discuss generalized lower record values and obtain recurrence relation for single and product moments from inverted Gompertz distribution. Finally, in Section 5 we give a charaterization result for the inverted Gompertz distribution.

\section{Recurrence relation for single and product moments of generalized upper record values from Gompertz distribution}

A random variable $X$ is said to have a Gompertz distribution if its pdf is of the form

$$
f(x)=\beta e^{\alpha x} e^{\left[-\frac{\beta}{\alpha}\left(e^{\alpha x}-1\right)\right]}, \quad 0 \leqslant x<\infty, \quad \alpha, \beta>0 .
$$

The cdf corresponding to (2.1) is given by

$$
F(x)=1-e^{\left[-\frac{\beta}{\alpha}\left(e^{\alpha x}-1\right)\right]} .
$$

It can be seen that

$$
f(x)=[\beta+\alpha\{-\ln [\bar{F}(x)]\}] \bar{F}(x),
$$

where $\bar{F}(x)=1-F(x)$. 
Let $\left\{Y_{m}^{(k)}, m \geqslant 1\right\}$ where $Y_{m}^{(k)}=X_{U_{m}^{(k)}: U_{m}^{(k)}+k-1}$ be a sequence of generalized upper record values from (2.1). Then the pdf of $Y_{m}^{(k)}, m \geqslant 1$ is given by [see, Dziubdziela and Kopocinski, 1976]

$$
f_{Y_{m}^{(k)}}(x)=\frac{k^{m}}{(m-1) !}[-\ln \bar{F}(x)]^{m-1}[\bar{F}(x)]^{k-1} f(x)
$$

and the joint density function of $Y_{m}^{(k)}$ and $Y_{t}^{(k)}, 1 \leqslant m<t, t \geqslant 2$ is given by

$$
\begin{aligned}
f_{Y_{m}^{(k)}, Y_{t}^{(k)}}(x, y) & =\frac{k^{t}}{(m-1) !(t-m-1) !}[\ln \bar{F}(x)-\ln \bar{F}(y)]^{t-m-1} \\
& \times[-\ln \bar{F}(x)]^{m-1} \frac{f(x)}{\bar{F}(x)}[\bar{F}(y)]^{k-1} f(y), \quad x<y .
\end{aligned}
$$

Theorem 2.1. Fix a positive integer $k \geqslant 1$. For $m \geqslant 1$ and $r=0,1,2, \ldots$

$$
\mu_{m+1 ; k}^{(r+1)}=\mu_{m ; k}^{(r+1)}-\frac{\beta k}{m \alpha}\left(\mu_{m ; k}^{(r+1)}-\mu_{m-1 ; k}^{(r+1)}\right)+\frac{(r+1)}{m \alpha} \mu_{m ; k}^{(r)} .
$$

Proof. For $m \geqslant 1$ and $r=0,1,2, \ldots$, we have from (2.3) and (2.4)

$$
\begin{aligned}
\mu_{m ; k}^{(r)} & =\frac{\beta k^{m}}{(m-1) !} \int_{0}^{\infty} x^{r}[-\ln \bar{F}(x)]^{m-1}[\bar{F}(x)]^{k} d x \\
& +\frac{\alpha k^{m}}{(m-1) !} \int_{0}^{\infty} x^{r}[-\ln \bar{F}(x)]^{m}[\bar{F}(x)]^{k} d x .
\end{aligned}
$$

Now, integrating by parts treating $x^{r}$ for integration and the rest of the integrand for differentiation and simplifying we get

$$
\mu_{m ; k}^{(r)}=\frac{\beta k}{(r+1)} \mu_{m ; k}^{(r+1)}-\frac{\beta k}{(r+1)} \mu_{m-1 ; k}^{(r+1)}+\frac{m \alpha}{(r+1)} \mu_{m+1 ; k}^{(r+1)}-\frac{m \alpha}{(r+1)} \mu_{m ; k}^{(r+1)} .
$$

Now, rearranging the terms we get

$$
\mu_{m+1 ; k}^{(r+1)}=\mu_{m ; k}^{(r+1)}-\frac{\beta k}{m \alpha}\left(\mu_{m ; k}^{(r+1)}-\mu_{m-1 ; k}^{(r+1)}\right)+\frac{(r+1)}{m \alpha} \mu_{m ; k}^{(r)},
$$

which is relation (2.6).

Remark 2.1. For $k=1$, we deduce the relation for single moments of upper record values established by Khan and Zia (2009).

Theorem 2.2. For $1 \leqslant m \leqslant t-2$ and $r, s=0,1,2, \ldots$

$$
\mu_{m, t ; k}^{(r+1, s)}=\mu_{m+1, t ; k}^{(r+1, s)}+\frac{\beta k}{m \alpha}\left(\mu_{m, t-1 ; k}^{(r+1, s)}-\mu_{m-1, t-1 ; k}^{(r+1, s)}\right)-\frac{(r+1)}{m \alpha} \mu_{m, t ; k}^{(r, s)}
$$

and for $m \geqslant 1, r, s=0,1,2, \ldots$

$$
\mu_{m, m+1 ; k}^{(r+1, s)}=\mu_{m+1 ; k}^{(s+r+1)}+\frac{\beta k}{m \alpha}\left(\mu_{m ; k}^{(s+r+1)}-\mu_{m-1, m ; k}^{(r+1, s)}\right)-\frac{(r+1)}{m \alpha} \mu_{m, m+1 ; k}^{(r, s)} .
$$


Proof. From (2.5), for $1 \leqslant m \leqslant t-1$ and $r, s=0,1,2, \ldots$, we have

$$
\mu_{m, t ; k}^{(r, s)}=\frac{k^{t}}{(m-1) !(t-m-1) !} \int_{0}^{\infty} y^{s}[\bar{F}(y)]^{k-1} f(y) I(y) d y
$$

where,

$$
I(y)=\int_{0}^{y} x^{r}[\ln \bar{F}(x)-\ln \bar{F}(y)]^{t-m-1}[-\ln \bar{F}(x)]^{m-1} \frac{f(x)}{\bar{F}(x)} d x .
$$

Using (2.3) in (2.10) we get

$$
\begin{aligned}
I(y) & =\beta \int_{0}^{y} x^{r}[\ln \bar{F}(x)-\ln \bar{F}(y)]^{t-m-1}[-\ln \bar{F}(x)]^{m-1} d x \\
& +\alpha \int_{0}^{y} x^{r}[\ln \bar{F}(x)-\ln \bar{F}(y)]^{t-m-1}[-\ln \bar{F}(x)]^{m} d x .
\end{aligned}
$$

Integrating (2.11) by parts, treating $x^{r}$ for integration and the rest of the integrand for differentiation, we get for $t \geqslant m+2$,

$$
\begin{aligned}
I(y) & =\frac{\beta(t-m-1)}{(r+1)} \int_{0}^{y} x^{r+1}[\ln \bar{F}(x)-\ln \bar{F}(y)]^{t-m-2} \frac{f(x)}{\bar{F}(x)}[-\ln \bar{F}(x)]^{m-1} d x \\
& -\frac{\beta(m-1)}{(r+1)} \int_{0}^{y} x^{r+1}[\ln \bar{F}(x)-\ln \bar{F}(y)]^{t-m-1} \frac{f(x)}{\bar{F}(x)}[-\ln \bar{F}(x)]^{m-2} d x \\
& +\frac{\alpha(t-m-1)}{(r+1)} \int_{0}^{y} x^{r+1}[\ln \bar{F}(x)-\ln \bar{F}(y)]^{t-m-2} \frac{f(x)}{\bar{F}(x)}[-\ln \bar{F}(x)]^{m} d x \\
& -\frac{\alpha m}{(r+1)} \int_{0}^{y} x^{r+1}[\ln \bar{F}(x)-\ln \bar{F}(y)]^{t-m-1} \frac{f(x)}{\bar{F}(x)}[-\ln \bar{F}(x)]^{m-1} d x .
\end{aligned}
$$

Substituting (2.12) in (2.9) and on further simplification we get (2.7).

Further, for $t=m+1$ we have

$$
\begin{aligned}
I(y) & =\frac{\beta}{(r+1)}\left[y^{r+1}[-\ln \bar{F}(y)]^{m-1}-(m-1) \int_{0}^{y} x^{r+1}[-\ln \bar{F}(x)]^{m-2} \frac{f(x)}{\bar{F}(x)} d x\right] \\
& +\frac{\alpha}{(r+1)}\left[y^{r+1}[-\ln \bar{F}(y)]^{m}-m \int_{0}^{y} x^{r+1}[-\ln \bar{F}(x)]^{m-1} \frac{f(x)}{\bar{F}(x)} d x\right] .
\end{aligned}
$$

Substituting (2.13) in (2.9) and simplifying we get (2.8).

Remark 2.2. For $k=1$, we deduce the relation for product moments of upper record values established by Khan and Zia (2009).

\section{Characterization result}

Now we obtain the characterization of Gompertz distribution using conditional expectation of function of generalized upper record values. First we describe the conditional distribution of a generalized upper record value $Y_{t}^{(k)}$ given $Y_{m}^{(k)}=x$ for $m<t$. We make use of this conditional distribution to obtain a characterization property of Gompertz distribution. The joint pdf of $Y_{m}^{(k)}$ and $Y_{t}^{(k)}$ is given 
in (2.5) and the marginal distribution of $Y_{m}^{(k)}$ is given by (2.4). If we write $h(y \mid x)$ to denote this conditional density, then the conditional pdf of $Y_{t}^{(k)}$ given $Y_{m}^{(k)}=x, 1 \leqslant m<t$ is given by

$$
h(y \mid x)=\frac{k^{t-m}}{(t-m-1) ![\bar{F}(x)]^{k}}[\ln \bar{F}(x)-\ln \bar{F}(y)]^{t-m-1}[\bar{F}(y)]^{k-1} f(y) .
$$

Clearly $h(y \mid x)$ is distributed as the pdf of the $(t-m)^{\text {th }}$ generalized upper record value arising from the distribution truncated on the left at $x$. Thus we conclude that the conditional pdf of generalized upper record values also shows a property similar to that of conditional pdf of an order statistic arising from an absolutely continuous distribution given the value of a lower order statistic.

In particular, the conditional pdf of $Y_{m+1}^{(k)}$ given $Y_{m}^{(k)}=x$, is given by

$$
h(y \mid x)=\frac{k[\bar{F}(y)]^{k-1} f(y)}{[\bar{F}(x)]^{k}}, \quad y \geqslant x .
$$

Theorem 3.1. Let $X$ be an absolutely continuous r.v. with $p d f f(x)$ and $c d f F(x)$. Then $X$ follows Gompertz distribution with $F(x)=1-e^{-\frac{\beta}{\alpha}\left(e^{\alpha x}-1\right)}, \alpha, \beta>0,0<x<\infty$ if and only if

$$
E\left[e^{-e^{\alpha Y_{m+1}^{(k)}}} \mid Y_{m}^{(k)}=x\right]=\frac{\beta k}{(\alpha+\beta k)} e^{-e^{\alpha x}}
$$

Proof. From (3.2) we get,

$$
E\left[e^{-e^{\alpha Y_{m+1}^{(k)}}} \mid Y_{m}^{(k)}=x\right]=\frac{k}{[\bar{F}(x)]^{k}} \int_{x}^{\infty} e^{-e^{\alpha y}}[\bar{F}(y)]^{k-1} f(y) d y .
$$

Now, using (2.1) and (2.2) in (3.4) the proof of the necessary part follows.

Conversely, assume that (3.3) holds. Then

$$
\int_{x}^{\infty} e^{-e^{\alpha y}}[\bar{F}(y)]^{k-1} f(y) d y=\frac{\beta}{(\alpha+\beta k)} e^{-e^{\alpha x}}[\bar{F}(x)]^{k} .
$$

Differentiating both sides of (3.5) and simplifying we get,

$$
-\frac{d}{d x} \ln \bar{F}(x)=\beta e^{\alpha x}
$$

which on further simplification leads to, $\bar{F}(x)=e^{-\frac{\beta}{\alpha}\left(e^{\alpha x}-1\right)}$ and this proves the theorem.

\section{Recurrence relation for single and product moments of generalized lower record values from inverted Gompertz distribution}

A r.v. $X$ is said to have an inverted Gompertz distribution if its pdf is of the form

$$
f(x)=\frac{\beta}{x^{2}} e^{-\frac{\beta}{\alpha}\left(e^{\frac{\alpha}{x}}-1\right)} e^{\frac{\alpha}{x}}, \quad x \geqslant 0, \quad \alpha, \beta>0
$$

and its cdf is of the form

$$
F(x)=e^{-\frac{\beta}{\alpha}\left(e^{\frac{\alpha}{x}}-1\right)} .
$$

It should be noted that if $Y$ follows a Gompertz distribution with pdf (2.1), then $X=1 / Y$ follows the inverted Gompertz distribution defined by the pdf (4.1). For an inverted Gompertz distribution 
$f(x)$ and $F(x)$ are connected by the relation

$$
x^{2} f(x)=[\beta+\alpha(-\ln F(x))] F(x) .
$$

Let $\left\{X_{n}, n \geqslant 1\right\}$ be a sequence of iid random variables as defined in Section 2. For a fixed $k \geqslant 1$, we define the sequence $\left\{L_{m}^{(k)}, m \geqslant 1\right\}$ of $k$-th lower record times of $\left\{X_{n}, n \geqslant 1\right\}$ (as introduced by Pawlas and Szynal, 1998) as follows:

$$
L_{1}^{(k)}=1, \quad L_{m+1}^{(k)}=\min \left\{j>L_{m}^{(k)}: X_{k: L_{m}^{(k)}+k-1}>X_{k: j+k-1}\right\} .
$$

For $k=1$, we write $L_{m}^{(1)}=L_{m}$ which are lower record times of $\left\{X_{n}, n \geqslant 1\right\}$.

The sequence $\left\{Z_{m}^{(k)}, m \geqslant 1\right\}$ where $Z_{m}^{(k)}=X_{k: L_{m}^{(k)}+k-1}$ is called the sequence of generalized lower record values or $k$-th lower record values of $\left\{X_{n}, n \geqslant 1\right\}$. For convenience we shall also take $Z_{0}^{(k)}=0$. For $k=1$, we have $Z_{m}^{(1)}=X_{L_{m}}, m \geqslant 1$, which defines the usual sequence of lower record values of $\left\{X_{n}, n \geqslant 1\right\}$. We shall define,

$$
\begin{array}{ll}
v_{m ; k}^{(r)}=E\left[\left(Z_{m}^{(k)}\right)^{r}\right], & r, m=1,2, \ldots \\
v_{m, t ; k}^{(r, s)}=E\left[\left(Z_{m}^{(k)}\right)^{r}\left(Z_{t}^{(k)}\right)^{s}\right], & 1 \leqslant m \leqslant t-1, r, s=1,2, \ldots \\
v_{m, t ; k}^{(r, 0)}=E\left[\left(Z_{m}^{(k)}\right)^{r}\right]=v_{m ; k}^{(r)}, & 1 \leqslant m \leqslant t-1, r=1,2, \ldots \\
v_{m, t ; k}^{(0, s)}=E\left[\left(Z_{t}^{(k)}\right)^{s}\right]=v_{t ; k}^{(s)}, & 1 \leqslant m \leqslant t-1, \quad s=1,2, \ldots
\end{array}
$$

The pdf of $Z_{m}^{(k)}, m \geqslant 1$ is given by

$$
f_{Z_{m}^{(k)}}(x)=\frac{k^{m}}{(m-1) !}[-\ln F(x)]^{m-1}[F(x)]^{k-1} f(x)
$$

and the joint density function of $Z_{m}^{(k)}$ and $Z_{t}^{(k)}, 1 \leqslant m<t, t \geqslant 2$ is given by

$$
\begin{aligned}
f_{Z_{m}^{(k)}, Z_{t}^{(k)}}(x, y) & =\frac{k^{t}}{(m-1) !(t-m-1) !}[\ln F(x)-\ln F(y)]^{t-m-1} \\
& \times[-\ln F(x)]^{m-1} \frac{f(x)}{F(x)}[F(y)]^{k-1} f(y), \quad x>y .
\end{aligned}
$$

Theorem 4.1. Fix a positive integer $k \geqslant 1$. For $m \geqslant 1$ and $r=1,2, \ldots$

$$
v_{m+1 ; k}^{(r)}=v_{m ; k}^{(r)}+\frac{\beta k}{m \alpha}\left(v_{m-1 ; k}^{(r)}-v_{m ; k}^{(r)}\right)-\frac{r}{m \alpha} v_{m ; k}^{(r+1)} .
$$

Proof. For $m \geqslant 1$ and $r=1,2, \ldots$, we have from (4.3) and (4.4)

$$
\begin{aligned}
v_{m ; k}^{(r+1)} & =\frac{\beta k^{m}}{(m-1) !} \int_{0}^{\infty} x^{r-1}[-\ln F(x)]^{m-1}[F(x)]^{k-1} d x \\
& +\frac{\alpha k^{m}}{(m-1) !} \int_{0}^{\infty} x^{r-1}[-\ln F(x)]^{m}[F(x)]^{k} d x .
\end{aligned}
$$

Now, integrating by parts treating $x^{r-1}$ for integration and the rest of the integrand for differentiation and simplifying we get relation (4.6). 
Theorem 4.2. For $1 \leqslant m \leqslant t-2$ and $r, s=1,2, \ldots$

$$
r v_{m, t ; k}^{(r+1, s)}=\beta k\left(v_{m-1, t-1 ; k}^{(r, s)}-v_{m, t-1 ; k}^{(r, s)}\right)+m \alpha\left(v_{m, t ; k}^{(r, s)}-v_{m+1, t ; k}^{(r, s)}\right)
$$

and for $m \geqslant 1, r, s=1,2, \ldots$

$$
r v_{m, m+1 ; k}^{(r+1, s)}=\beta k\left(v_{m-1, m ; k}^{(r, s)}-v_{m ; k}^{(s+r)}\right)+m \alpha\left(v_{m, m+1 ; k}^{(r, s)}-v_{m+1 ; k}^{(s+r)}\right) .
$$

Proof. The proof follows exactly in the same manner as in Theorem 2.2 and hence omitted.

\section{Characterization result for inverted Gompertz distribution}

Here we obtain a characterization property of inverted Gompertz distribution based on conditional expectation of function of generalized lower record values. First we consider the conditional distribution of a generalized lower record value $Z_{t}^{(k)}$ given $Z_{m}^{(k)}=x$ for $m<t$. The joint pdf of $Z_{m}^{(k)}$ and $Z_{t}^{(k)}$ is given in (4.5) and the marginal distribution of $Z_{m}^{(k)}$ is given by (4.4). If we write $g(y \mid x)$ to denote this conditional density, then the conditional pdf of $Z_{t}^{(k)}$ given $Z_{m}^{(k)}=x, 1 \leqslant m<t$ is given by

$$
g(y \mid x)=\frac{k^{t-m}}{(t-m-1) ![F(x)]^{k}}[\ln F(x)-\ln F(y)]^{t-m-1}[F(y)]^{k-1} f(y) .
$$

Clearly $g(y \mid x)$ is distributed as the pdf of the $(t-m) t h$ generalized lower record value arising from the distribution truncated on the right at $x$. Thus we conclude that the conditional pdf of generalized lower record values also shows a property similar to that of conditional pdf of an order statistic arising from an absolutely continuous distribution given the value of a higher order statistic.

In particular, the conditional pdf of $Z_{m+1}^{(k)}$ given $Z_{m}^{(k)}=x$, is given by

$$
g(y \mid x)=\frac{k[F(y)]^{k-1} f(y)}{[F(x)]^{k}} .
$$

Theorem 5.1. Let $X$ be an absolutely continuous r.v. with $p d f f(x)$ and $c d f F(x)$. Then $X$ follows an inverted Gompertz distribution with $F(x)=e^{-\frac{\beta}{\alpha}\left(e^{\frac{\alpha}{x}}-1\right)}$ if and only if

$$
E\left[e^{-e^{\alpha / z_{m+1}^{(k)}}} \mid Z_{m}^{(k)}=x\right]=\frac{\beta k}{(\alpha+\beta k)} e^{-e^{\frac{\alpha}{x}}} .
$$

Proof. Using (5.2), the necessary part follows by direct computation.

Conversely, assume that (5.3) holds. Then

$$
\int_{0}^{x} e^{-e^{\frac{\alpha}{y}}}[F(y)]^{k-1} f(y) d y=\frac{\beta}{(\alpha+\beta k)} e^{-e^{\frac{\alpha}{x}}}[F(x)]^{k} .
$$

Differentiating both sides of (5.4) and simplifying we get,

$$
\frac{d}{d x} \ln F(x)=\frac{\beta e^{\frac{\alpha}{x}}}{x^{2}}
$$

which on further simplification leads to, $F(x)=e^{-\frac{\beta}{\alpha}\left(e^{\frac{\alpha}{x}}-1\right)}$. 


\section{Acknowledgements}

The authors acknowledge their gratefulness to the learned referee for their valuable comments and suggestions.

\section{References}

[1] Ahsanullah, M. (1988). Introduction to Record Statistics. Ginn Press, Needham Heights, Massachusetts.

[2] Ahsanullah, M. (1995). Record Statistics. Nova Science Publishers, Commack, New York.

[3] Ahuja, J.C. and Nash, S.W. (1967). The generalized Gompertz-Verhulst family of distributions. Sankhya A, 29, 141-156.

[4] Al-Hussaini, E.K., Al-Dayian, G.R. and Adham, S.A. (2000). On finite mixture of two-component Gompertz lifetime model. J. Statist. Comput. Simul., 67, 1-15.

[5] Arnold, B.C., Balakrishnan, N. and Nagaraja, H.N. (1998). Records. John Wiley and Sons, New York.

[6] Chandler, K.N. (1952). The distribution and frequency of record values. Journal of Royal Statistical Society. Ser. B, 14, 220-228.

[7] Dzuibdziela, W. and Kopocinski, B. (1976). Limiting properties of the $k$ th record values. Zastosowania Mathematyki, 15, 187-190.

[8] Gompertz, B. (1825). On the nature of the function expressive of the law of human mortality and on a new model of determining the value of life contingencies. Philos. Trans. Roy. Soc. Lond., 115, 513-585.

[9] Khan, R.U. and Zia, B. (2009). Recurrence relations for single and product moments of record values from Gompertz distribution and a characterization. World Applied Sciences Journal, 7, 1331-1334.

[10] Marshall, A.W. and Olkin, I. (2007). Life Distributions: Structure of Non-parametric, Semiparametric and Parametric Families. Springer-Science, New York.

[11] Pawlas, P. and Szynal, D. (1998). Recurrence relations for single and product moment of $k$-th record values from exponential and Gumbel distribution and a characterization. Journal of Applied Statistical Science, 8, 53-62. 